\title{
BMJ Open ITM support for patients with chronic respiratory and cardiovascular diseases: a protocol for a randomised controlled trial
}

\author{
Julie Redfern, ${ }^{01,2}$ Karice Hyun, ${ }^{1}$ Anna Singleton, ${ }^{1}$ Nashid Hafiz, ${ }^{1}$ \\ Rebecca Raeside, ${ }^{1}$ Lissa Spencer, ${ }^{3}$ Bridie Carr, ${ }^{4}$ Ian Caterson, ${ }^{5}$ John Cullen, ${ }^{6}$ \\ Cate Ferry, ${ }^{7}$ Karla Santo, ${ }^{8,9}$ Alison Hayes, ${ }^{10}$ Regina W M Leung, ${ }^{11}$ \\ Simon Raadsma, ${ }^{12}$ Jessica Swinbourne, ${ }^{13}$ Jin G Cho, ${ }^{14}$ Meredith King, ${ }^{15}$ \\ Mary Roberts, ${ }^{16}$ Cindy Kok, ${ }^{14}$ Christine Jenkins, ${ }^{17}$ Clara Chow ${ }^{1,16}$
}

To cite: Redfern J, Hyun K, Singleton A, et al. ITM support for patients with chronic respiratory and cardiovascular diseases: a protocol for a randomised controlled trial. BMJ Open 2019;9:e023863. doi:10.1136/ bmjopen-2018-023863

- Prepublication history for this paper is available online. To view these files, please visit the journal online (http://dx.doi. org/10.1136/bmjopen-2017023863).

Received 14 May 2018 Revised 12 January 2019 Accepted 15 January 2019

Check for updates

(C) Author(s) (or their employer(s)) 2019. Re-use permitted under CC BY-NC. No commercial re-use. See rights and permissions. Published by BMJ.

For numbered affiliations see end of article.

Correspondence to Professor Julie Redfern; julie.redfern@sydney.edu.au

\section{ABSTRACT}

Introduction Simple and scalable strategies are needed to improve 'out-of-hospital' support and management for people living with cardiovascular disease (CVD) and respiratory disease. Text messaging via mobile phones has been shown to be effective in helping promote lifestyle change and is supported by quantitative and qualitative evidence. The aim of this study is to test the effectiveness and implementation of a 6-month text messaging support programme for people with CVD and respiratory disease as an addition to cardiac and pulmonary outpatient rehabilitation.

Methods and analysis Pragmatic randomised controlled trial $(n=310)$ to test the effectiveness of a 6 -month text message support programme on clinical outcomes in people with CVD and chronic respiratory disease who are attending outpatient cardiac and pulmonary rehabilitation. The study includes a nested process evaluation to inform scalability and implementation across settings. The intervention group will receive a text message support programme comprising five messages per week for 26 weeks and the control group will continue with standard care. The primary outcome is exercise capacity $(6 \mathrm{~min}$ walk distance). Secondary outcomes include clinical measures (proportion of people meeting the Australian guideline-recommended blood pressure and cholesterol targets), lifestyle outcomes (smoking rates, achievement of national guidelines for nutrition and physical activity), quality of life, mood (Hospital Anxiety and Depression Scale), medication adherence and attendance at and completion of rehabilitation.

Ethics and dissemination Primary ethics approval was received from the Sydney Local Health District Hospital Human Research Ethics Committee and associated Governance committees at sites. Results will be disseminated via the usual scientific forums including peer-reviewed publications and presentations at international conferences. At its conclusion, the study will determine the effectiveness and implementation of a simple programme that aims to improve health outcomes and attendance at rehabilitation for people with CVD and chronic respiratory disease.
Strengths and limitations of this study

- This study is a randomised controlled clinical trial and will determine whether the text message support programme can be delivered within existing cardiac and pulmonary rehabilitation.

- The study will assess objective measures to determine whether an evidence-based text message intervention can be successfully delivered to patients with both cardiac and chronic respiratory diseases.

- The study design has a pragmatic and implementation focus and includes a process evaluation to inform translation.

- The study is limited to a relatively small number of participants from six sites across Sydney, Australia.

Trial registration number ACTRN12616001167459

\section{INTRODUCTION}

Noncommunicable diseases (NCDs), also known as chronic diseases, are the leading cause of death globally, accounting for $68 \%$ of the total deaths in 2012. ${ }^{2}$ Cardiovascular disease (CVD) and chronic respiratory disease are two of the major NCDs associated with preventable hospital admissions ${ }^{1}$ and have a negative impact on quality of life and healthcare costs. ${ }^{3-5}$ Approximately half of the patients with coronary disease have had prior events, ${ }^{6}$ only the minority take appropriate medicines, receive lifestyle advice and a referral to rehabilitation at the point of discharge. ${ }^{7-11}$ Similarly, moderate-to-severe chronic obstructive pulmonary disease (COPD) occurs in approximately 65 million people worldwide and it is predicted that COPD will become the third leading cause of death worldwide in 2030. ${ }^{12} 13$ Overall, we 
are faced with an environment of increasing chronic disease burden coupled with an ageing population and finite resources. Therefore, simple strategies to improve 'out-of-hospital' management and support are needed.

Chronic disease management programmes typically offer face-to-face group exercise and education sessions as part of 'rehabilitation' with the aim to improve health-related behaviour and symptom management. ${ }^{14}$ These programmes are effective in reducing hospital readmissions, improve clinical parameters, adherence to treatments and quality of life ${ }^{13-16}$ However, engagement, participation and maintenance of involvement are suboptimal with less than $30 \%$ of those eligible attending and completing. ${ }^{17} 18$ The emerging availability of new technologies provides an opportunity to support self-management and increase participation in existing programmes coupled with the availability of ongoing support. Worldwide, more than 4.7 billion people own mobile phones (with mobile phone penetration in Australia is $>80 \%$ and $68 \%$ own smartphones), ${ }^{19}$ and text messaging has become a popular and simple way to communicate in a low cost and unobtrusive way. Randomised controlled trials (RCTs) have demonstrated the effectiveness of text messaging to promote smoking cessation, ${ }^{20}$ weight loss, ${ }^{21}$ physical activity ${ }^{22}$ and blood pressure (BP) lowering. ${ }^{23}$ The Tobacco, Exercise and Diet Messages (TEXT ME) trial was a recent single-blind RCT of a lifestyle-focused text messaging support programme delivered for 6 months. ${ }^{24}$ The TEXT ME study showed that, compared with control, at 6 months, the intervention group achieved a lower low-density lipoprotein cholesterol, systolic blood pressure (SBP), body mass index (BMI), smoking rates and a higher physical activity level. ${ }^{25}$ The programme was also found to be cost-effective ${ }^{26}$ and engaging, useful and easy to understand by patients. ${ }^{27}$

The aim of this present study is to evaluate the effectiveness of a post-discharge text message support programme, compared with usual care, for patients with chronic disease (CVD and/or chronic respiratory disease) attending cardiac and pulmonary rehabilitation programmes. We also aim to explore factors that enhance participation in subpopulations (eg, different socioeconomic backgrounds, older/younger, gender, primary language spoken) and determine the acceptability and utility of implementing the text messaging self-management support programme among participants and staff across participating sites.

\section{METHODS AND ANALYSIS \\ Study design}

Pragmatic, multicentre, single-blind, randomised controlled implementation trial across at least six rehabilitation clinics (with embedded qualitative analysis) in Australia (figure 1). Approximately 310 patients attending either the cardiac or pulmonary rehabilitation will be randomised (3:1) to either a 6 -month text message support programme (intervention) or standard care (control). Clinical, behavioural and quality of life data will be collected at face-to-face assessments at baseline and 6 months. Written and informed consent will be obtained from all participants and the study will follow the Consolidated Standards of Reporting Trial guidelines. ${ }^{28}$ The study's first patient was recruited in May 2017 and final follow-ups are anticipated for completion in by June 2019.

\section{Patient and public involvement}

This study design and question was developed following extensive consultation with patients involved in our previous research and focus groups. ${ }^{27}$ These patients all had a previous diagnosis of cardiac and chronic respiratory diseases and were between the ages of 40 and 80 years. Patient feedback suggested that the post-discharge period was an important time for influencing behaviour and that they valued receipt of the intervention and hence the randomisation ratio was set to enable more participants to receive the intervention. Patient involvement also drove the content development for the intervention itself. This information and feedback was collected by surveys and focus groups with patients where they prioritised information from credible sources, content that was practical and they specifically requested messages be sent four to five times per week and that the content be semipersonalised. ${ }^{27}$ In terms of outcomes, meaningful and functional outcomes such as walking distance and quality of life were selected to align with goals of patients with cardiac and respiratory diseases. Patients participated via being active participants in the content development workshops and also in completing surveys. Therefore, patients were involved in design of the intervention content, message delivery schedule and study outcomes. As part of the current study, we will collect process data that will include assessment of patient satisfaction, rating of perceived usefulness and an opportunity to provide feedback about the programme and its delivery. At the conclusion of the study, we will disseminate the results among consumer and patient organisations such as the National heart Foundation and the Lung Foundation of Australia.

\section{Randomisation and allocation concealment}

Participants will be randomised to either usual standard care (control) or text messaging programme (intervention) using a computerised randomisation programme. Randomisation (stratified according to respiratory or cardiac rehabilitation) will be conducted independently using a central computer-based randomisation service and the random allocation sequence will be concealed from study personnel and will take place after collection of baseline data. Given the study is a pragmatic implementation trial, participants will be individually randomised at a ratio of 3:1 (intervention:control) to enable more participants to receive the text message programme. Study personnel taking follow-up measures will also be blinded to parallel group assignment. Participants will be 


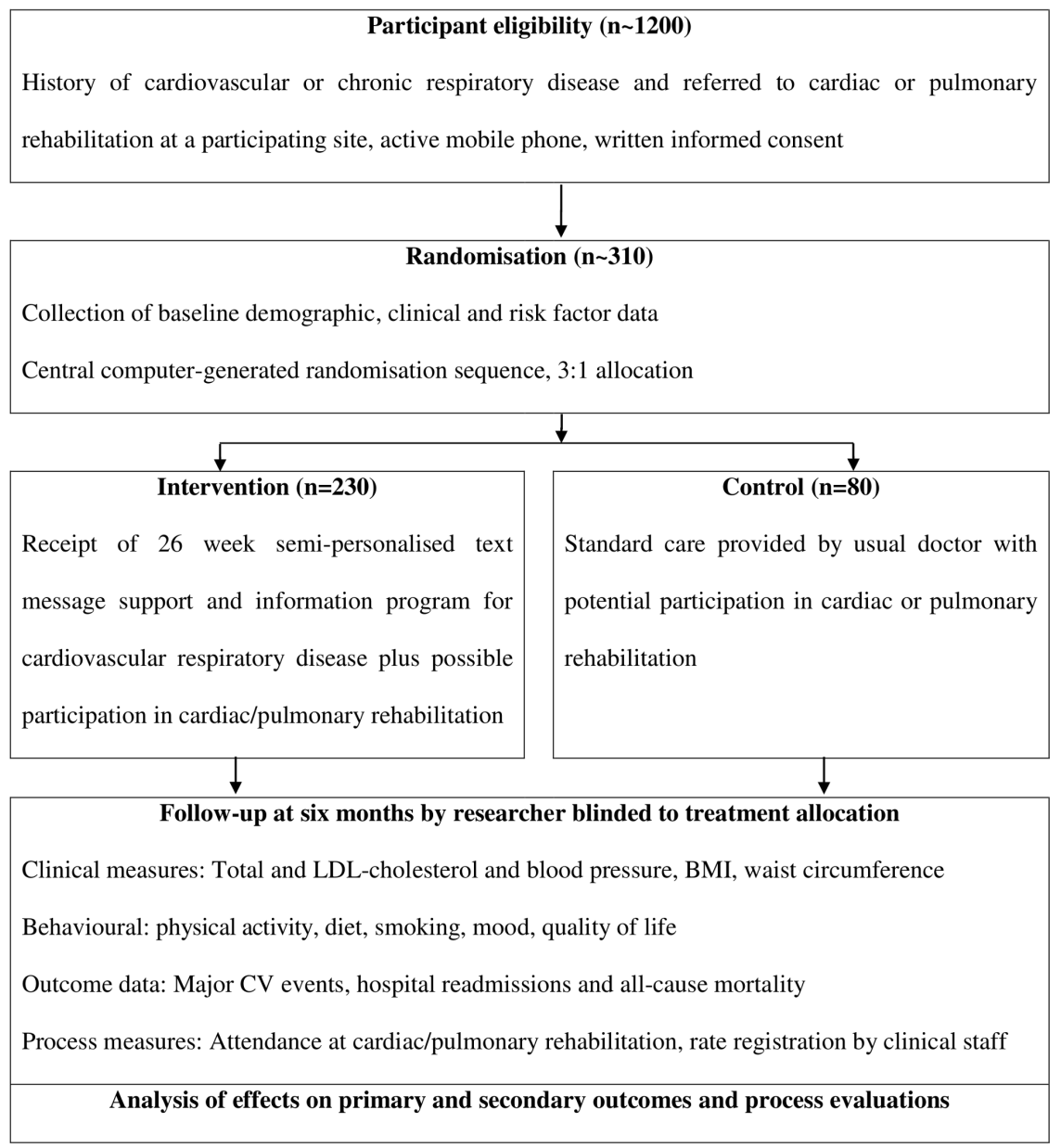

Figure 1 Study flow diagram. LDL, low-density lipoprotein; BMI, body mass index; CV, cardiovascular.

encouraged to not discuss whether they are receiving or not the text messages during their follow-up visit.

\section{Participant eligibility}

Participants will be recruited from participating hospital-based, outpatient rehabilitation programmes across Sydney.

Inclusion criteria are as follows:

i. Adults aged above 18 years;

ii. Have an active mobile phone that is capable of receiving text messages.

iii. Have a medical history of CVD, including coronary heart disease, cardiomyopathy, peripheral arterial disease, stroke and/or;

iv. History of chronic respiratory disease, including COPD, chronic bronchitis, emphysema, chronic asthma and bronchiectasis.

\section{Exclusion criteria are as follows}

i. Unlikely to comply with the demands of the study for 6 months;

ii. Insufficient English language skills to provide written and informed consent.

\section{Recruitment}

Potential patients will be identified by clinical rehabilitation staff at participating teaching hospitals and invited to participate. Participation (or not) will not impact on the ability to participate in the rehabilitation programme but will be offered as an additional support for patients if they volunteer and are allocated to the intervention group. Clinical staff will obtain consent from those willing to volunteer along with baseline data as part of routine clinical assessment for cardiac or pulmonary rehabilitation. Once recruited and baseline data are collected, clinical staff (where possible) will complete a simple web-based registration process that randomises the participant and commences the messaging programme for those allocated to the intervention group. If time prevents a clinician completing the online registration, a member of the research team with clinical experience will complete the required online form. A research assistant, blinded to group allocation, will then enter the collected data into a secure online database. Process data will be kept relating to these procedures to inform capacity and time needed for implementation of the programme. 


\section{Box 1 Example text messages sent to intervention group}

Patients participating in pulmonary rehabilitationPatients participating in pulmonary rehabilitation

- Pulmonary rehabilitation often includes medically supervised exercise and education to help you manage COPD and stay active. Ask your GP for a referral.

- Ask your GP or specialist to write you a COPD action plan to help you know when you should increase your medications and when you should go to the hospital.

- Hi (NAME), having emergency antibiotics at home allows you to treat flare ups early. Talk to your GP to see if it is an option for you.

- Eating a balanced diet and maintaining a healthy weight are important especially when you have a lung disease.

- When you are feeling breathless, follow the three Ps, Pause, Position and Pursed lip breathing (stop, lean forward and breath slowly).

Patients participating in cardiac rehabilitationPatients participating in cardiac rehabilitation

- (NAME), try identifying the triggers that make you want a cigarette and plan to avoid them.

- Did you know $90 \%$ of people do not eat the recommended daily intake of vegetables (five serves a day)?

- Hi (NAME), do not forget physical activity is good for you! It reduces your risk of diabetes, heart attack, stroke and their complications.

- Walking is cheap. It can be done almost anywhere. All you need is comfortable shoes and clothing.

- Studies show that stress, worry and loneliness can increase the risk of heart disease. Please talk to a health professional if you need help.

COPD, chronic pulmonary obstructive disease; GP, general practitioner

\section{Intervention group}

The intervention group will receive a 26-week text message programme in addition to cardiac or pulmonary rehabilitation. All participants randomised to the intervention group will receive five messages per week at random times of the day and on random days of the week for the duration of the programme. This approach has been chosen in accordance with our previous evidence-based text message quantitative and qualitative research. ${ }^{25-27}$ Participants will be encouraged not to reply to messages as the programme does not have the capacity for two-way communication with researchers or clinicians. However, all replies will be monitored for safety using the software programme and a centrally located research assistant with a clinical background. If participants wish to withdraw from the programme they will be asked to send a reply message saying 'stop' and this will activate a process of review and potential withdrawal from the study. The messages will be semipersonalised messages where some contain the participant's preferred name and are tailored for individual circumstances and preferences (eg, non-smoker, vegetarian, physical activity level). Example messages are provided in box 1 . The messages will be sent from a centralised web-based platform that provides simple registration and automated message delivery based on the team's previously published system that was also used in the TEXT ME study. ${ }^{25} 29$
The text messages are based on behavioural psychology and provide self-management support relating to medication adherence, attendance at chronic disease programmes and lifestyle change. The message programme content and structure was developed according to our previously published model. ${ }^{30}$ Some content for cardiac patients was based on our previous TEXT ME programme ${ }^{25}$ but the content for patients attending pulmonary rehabilitation was entirely new. This process involved literature review, surveys and focus groups with patients (cardiac and pulmonary diseases) recruited from chronic disease programmes. We used a patient-centred approach to expand our existing text message content used in previous research to ensure they contain content that is both evidence based and meaningful for patients. For CVD, the primary content areas for the text messages are general CVD health, smoking cessation, physical activity and nutrition. For chronic respiratory disease, the primary content areas are general respiratory health, physical activity, smoking cessation, symptom management and symptom monitoring. Both the CVD and respiratory message programmes also include content supporting medication adherence and participation in rehabilitation. Each week messages, without any repeats, are selected by the software system from the bank based on prespecified algorithms that ensure variety across the major focus areas for each condition.

\section{Control group}

Participants in the control group will receive ongoing standard care (including cardiac or pulmonary rehabilitation) without receiving the text message programme. All control participants will receive a single introductory messaging stating they are in the control group and that they will be contacted for follow-up at 6 months. In addition, all participants allocated to control will be offered the messaging programme after their 6-month follow-up. This will be optional for control participants and provided to them at no cost.

\section{Study outcomes}

The primary outcome of the study is the $6 \mathrm{~min}$ walk distance (box 1). The $6 \mathrm{~min}$ walk distance provides a commonly used assessment of exercise capacity ${ }^{31}$ that has been demonstrated to be both valid and reliable in cardiac and respiratory patient populations. Secondary outcomes (box 2) vary slightly between conditions (cardiac or respiratory) and include clinical measures (cholesterol, BP, BMI, waist circumference), lifestyle measures (nutrition, physical activity, smoking cessation), quality of life, depression and anxiety, medication adherence, hospital readmissions (self-report, hospital records) and attendance at and completion of allocated rehabilitation programme. We will also collect process measures such as satisfaction with the user interface for registration, participant satisfaction with the programme and content, rate and content of any reply messages received. 


\section{Box 2 Study outcomes measured at 6 months}

Primary outcomePrimary outcome

- Exercise capacity-6 min walk distance. ${ }^{31}$

Secondary outcomesSecondary outcomes

- Blood pressure - mean of two resting digital readings.

- Body mass index and waist circumference - height, weight, waist circumference. $^{32}$

- Point abstinence in smoking. ${ }^{33}$

- Depression and anxiety- Hospital Anxiety and Depression Scale. ${ }^{34}$

- Physical activity—Global Physical Activity Questionnaire. ${ }^{35}$

- Nutrition quality -Questions adapted from WHO STEPS Instrument. ${ }^{36}$

- Quality of life-SF-12 V2 (License number QM038623). ${ }^{37}$

- Medication adherence-Self report (validated through prescription data obtained via linkage with PBS).

- Hospital admissions - self report, hospital records and checked against linkage with Admitted Patient Data Collection for New South Wales.

- Impact of COPD on well-being-COPD Assessment Test (participants with respiratory disease only). ${ }^{38}$

Process outcomes (both respiratory and cardiac)Process outcomes (both respiratory and cardiac)

- Rate and content of text message responses (software analytics).

- Assessment of registration process (survey and interview with clinicians).

- Attendance at and completion of rehabilitation programme (programme records).

COPD, chronic obstructive pulmonary disease; CVD, cardiovascular disease; PBS, pharmaceutical benefits scheme; SF-12, short-form 12; WHO STEPS, WHO STEPwise approach to chronic disease risk factor surveillance.

These measures will be collected via a simple survey with likert responses.

\section{Data collection}

Participants will have outcomes assessed at baseline and 6 months. These outcomes align with data already collected within the usual chronic disease management programmes within the participating Local Health Districts (LHDs). Baseline data will be collected by clinical staff prior to randomisation and hence blinded to study group allocation. Given this is an implementation study, it is important to explore the potential to assess programme outcomes within existing practice and workloads. Follow-up data at 6 months will be collected (face to face or by telephone) by a research assistant blinded to treatment allocation. The central research team will manage and enter data into a secure online database for research purposes. We will also seek consent from participants to access Medicare Benefits Scheme (MBS) and Pharmaceutical Benefits Scheme (PBS) administrative data to determine health service utilisation and prescriptions for medications at 12 months after randomisation. Furthermore, we will seek participant consent to link hospital readmissions administrative with in New South Wales (via the Centre for Health Research Linkage) at 12 months after randomisation.

\section{Sample size and statistical analysis}

A sample size of approximately 310 will achieve above $90 \%$ power for both respiratory and cardiac subgroups to observe a clinically meaningful difference in $6 \mathrm{~min}$ walking distance in the intervention group compared with control. For respiratory patients, a mean difference (between intervention and control) of $30 \mathrm{~m}$ (SD 54.7) in 6 min walk distance is assumed to be clinically significant. ${ }^{39}$ Therefore, for $90 \%$ power, a sample size of 138 (103 in intervention and 35 in control) is needed and to allow for loss to follow-up the target sample size is 160 patients with respiratory disease. For cardiac patients, a mean difference (between intervention and control) of $23.3 \mathrm{~m}$ (SD 34.8) in $6 \mathrm{~min}$ walk distance is assumed to be clinically significant. ${ }^{40}$ Therefore, for $90 \%$ power, a sample size of 126 (94 in intervention and 32 in control) is needed and to allow for loss to follow-up the target sample size is 150 patients with cardiac disease. A sample size of 310 will also achieve $90 \%$ power to observe $20 \%$ increase in programme attendance in the intervention group compared with control.

The primary analysis will include all available participant data and will be performed at the end of the study after all data have been collected. The analysis of the primary and secondary outcomes will be done according to the intention-to-treat principle. For the primary analysis, analysis of covariance (ANCOVA), adjusting for disease (cardiac or respiratory), gender, site and the baseline measurement of the primary outcome will be used to examine differences between intervention and control at 6 months. For secondary analyses, ANCOVA will be used for continuous outcomes and logistic regression will be used for the categorical outcomes. Each model will be adjusted for disease (cardiac or respiratory), gender, site and the baseline measurement of the primary outcome. Planned subgroup analyses will be of cardiac versus respiratory disease, males versus females, older versus younger (60 as threshold), shorter distance at baseline versus longer distance at baseline for $6 \mathrm{~min}$ walk. A significance level of 0.05 will be used. All analysis will be undertaken using SAS V. 9.4 for Windows. To account for any missing data, we will perform a sensitivity analysis by repeating the main analysis using multiple imputation to include patients with missing outcomes.

\section{Process evaluation}

A process evaluation will be conducted comprising two parts: a semiquantitative survey and focus groups with participants, providers and staff involved in implementation. All intervention participants will also be administered a programme feedback questionnaire (paper or via telephone) to assess the tolerability of repeat text messages, asking which messages they remembered, liked or disliked, what they did with the messages (eg, kept them or deleted them immediately), their perceived utility of the text message and their opinion regarding the intrusiveness, timing and content suitability of the text messages. A subsample of patients, providers and 
staff (purposively selected based on maximum variability across sites and cardiac/respiratory disease) will also be invited to participate in a focus group or semistructured interviews to explore their perceptions of the utility and acceptability of the text messaging programme and its implementation. Focus groups will be facilitated by a trained professional and discussions will be digitally recorded and transcribed verbatim. Results will then be coded and interpreted to isolate key themes, contrasts and hierarchies, and to develop coding matrices based on a framework approach. In addition, logs will be kept to assess the time messages are sent and the proportion of text messages successfully delivered (eg, if mobile phone boxes are full, messages may not be able to be successfully delivered). A log will be kept of how many participants contact the study team, the reason for contact and the method used for contact (eg, telephone, email or text message). Implementation data will also be collected to determine how many patients were registered by clinical staff and what were the barriers and enablers to registration by those staff.

\section{DISCUSSION}

We propose a randomised controlled implementation study that tests the effectiveness of a text message support programme that educates, motivates and maintains a patient's connection with chronic care services through a semipersonalised text messages delivered for 6 months. The overall objective is to increase engagement and participation in chronic disease management programmes (namely cardiac and pulmonary rehabilitation) and to ultimately reduce hospital readmissions. The text messaging programme is simple, affordable and scalable. If successful, the text messaging programme would be applicable to many individuals living with chronic disease in Australia and beyond. Expected health outcomes include an increase in lifestyle modification, adherence to evidence based and prescribed medications and a reduction in hospital readmissions. We also anticipate an improvement in accessibility to chronic disease preventive care focusing on vulnerable/at risk families and communities such as those from lower socioeconomic backgrounds and patients with the greatest risk factors for disease. The study will also inform acceptability, functionality and future implementation into practice.

\section{Potential limitations}

This trial is only being conducted in one state of Australia and the sample size does not have power to detect changes in hospitalisation rates. Inclusion and exclusion criteria were intentionally broad given the focus on the study was implementation within the context of cardiac and pulmonary rehabilitation rather than effectiveness within strict clinical parameters and under strict control of external variables; however, this should be considered on balance in terms of potential generalisability and implementation. In addition, the collection of baseline data by clinicians is a potential limitation due to slight variation in environment and individual difference; however, standard measurement procedures will be followed and the primary analysis will be a comparison between groups at 6 months follow-up. The registration system is online; however, the user interface will require further refinement based on the results of the process evaluation.

\section{ETHICS AND DISSEMINATION}

The findings of this study will be disseminated via the usual scientific forums including peer-reviewed publications and presentations at international and national conferences. This study will adhere to the National Health and Medical Research Council ethical guidelines for human research. Protocol deviations will be reported to the associated ethics committees if required. Written informed consent will be obtained from all enrolled participants by the research staff. Additional individual informed consent for data linkage through MBS and PBS will also be obtained. Participants will be informed of their right to refuse to participate or to withdraw participation at any time without reprisal.

\section{Author affiliations}

${ }^{1}$ Westmead Applied Research Centre, Faculty of Medicine and Health, University of Sydney, Sydney, New South Wales, Australia

${ }^{2}$ Cardiovascular Division, The George Institute for Global Health, Sydney Medical School, University of Sydney, Sydney, New South Wales, Australia

${ }^{3}$ Department of Physiotherapy, Sydney Local Health District, Camperdown, New

South Wales, Australia

${ }^{4}$ NSW Agency for Clinical Innovation, Sydney, New South Wales, Australia ${ }^{5}$ Boden Institute, University of Sydney, University of Sydney, New South Wales, Australia

${ }^{6}$ Department of Geriatric Medicine, Concord Repatriation General Hospital, Sydney LHD, Sydney, New South Wales, Australia

${ }^{7}$ NSW Division, Heart Foundation, Sydney, New South Wales, Australia

${ }^{8}$ Cardiovascular Division, The George Institute for Global Health, Sydney, New South Wales, Australia

${ }^{9}$ Sydney Medical School, University of Sydney, Sydney, New South Wales, Australia

${ }^{10}$ School of Public Health, University of Sydney, Sydney, Australia

${ }^{11}$ Faculty of Health Sciences, University of Sydney, Sydney, New South Wales, Australia

${ }^{12}$ Behavioural Insights Unit, NSW Department of Premier and Cabinet, Sydney, New South Wales, Australia

${ }^{13}$ Boden Institute of Obesity, Nutrition, Exercise and Eating Disorders, University of Sydney, Sydney, New South Wales, Australia

${ }^{14}$ Westmead Clinical School, University of Sydney, Sydney, New South Wales, Australia

${ }^{15}$ Chronic Disease Community Rehabilitation Service, Northern Sydney Local Health District, Sydney, New South Wales, Australia

${ }^{16}$ Western Sydney Local Health District, Westmead, New South Wales, Australia

${ }^{17}$ Institute of Respiratory Medicine, Royal Prince Alfred Hospital, Sydney, New South Wales, Australia

Acknowledgements We thank all the staff and patients at participating hospitals for their support with design, recruitment and implementation of the study. We also thank Ms Rebecca MacNaughton for her role in the early stage of recruitment and Ms Nashid Hafiz for monitoring support. Also, we thank Mr Tony Barry, Dr Aravinda Thiagalingam and Ms Anu Indrawansa for their contributions and hard work with the software management system and monitoring.

Contributors JR and CC conceived the original concept of the study and the intervention. $\mathrm{CK}, \mathrm{RR}, \mathrm{NH}, \mathrm{KS}$ and $\mathrm{AS}$ supported details of recruitment and data collection. KH and JR performed the sample size calculations and will lead analysis 
of the results. JR and CK drafted the protocol. LS, BC, IC, JGC, CF, RWML, JS, SR, $\mathrm{AH}, \mathrm{MR}, \mathrm{MK}, \mathrm{JGC}$ and $\mathrm{CJ}$ supported message development and testing. All authors contributed to the scientific design of the study and the protocol development, and are involved in the implementation of the project and have read and approved the final manuscript.

Funding This work is supported by a National Heart Foundation 2015 NSW Cardiovascular Research Network Research Development Project Grant [ID: 101134]. JR is funded by a NHMRC Career Development Fellowship [APP1143538] CKC is funded by a Career Development Fellowship co-funded by the NHMRC and National Heart Foundation [APP1105447]

Competing interests None declared.

Patient consent for publication Not required.

Ethics approval Formal ethical approval has been obtained from Sydney Local Health District (Royal Prince Alfred Hospital zone), Human Research Ethics Committee (reference X16-0283) along with associated local Governance approvals at participating sites.

Provenance and peer review Not commissioned; externally peer reviewed.

Open access This is an open access article distributed in accordance with the Creative Commons Attribution Non Commercial (CC BY-NC 4.0) license, which permits others to distribute, remix, adapt, build upon this work non-commercially, and license their derivative works on different terms, provided the original work is properly cited, appropriate credit is given, any changes made indicated, and the use is non-commercial. See: http://creativecommons.org/licenses/by-nc/4.0/.

\section{REFERENCES}

1. World Health Organization. Non-communicable diseases, Fact sheet. 2017. http://www.who.int/mediacentre/factsheets/fs355/en/

2. World Health Organization. Global status report on noncommunicable diseases. Geneva: WHO, 2014.

3. Mittmann N, Kuramoto L, Seung SJ, et al. The cost of moderate and severe COPD exacerbations to the Canadian healthcare system. Respir Med 2008;102:413-21.

4. Scott IA. Chronic disease management: a primer for physicians. Intern Med J 2008;38:427-37.

5. Cournane S, Byrne D, O'Riordan D, et al. Chronic disabling diseaseimpact on outcomes and costs in emergency medical admissions. QJM 2015;108:387-96.

6. Briffa TG, Hobbs MS, Tonkin A, et al. Population trends of recurrent coronary heart disease event rates remain high. Circ Cardiovasc Qual Outcomes 2011;4:107-13.

7. Redfern J, Hyun K, Chew DP, et al. Prescription of secondary prevention medications, lifestyle advice, and referral to rehabilitation among acute coronary syndrome inpatients: results from a large prospective audit in Australia and New Zealand. Heart 2014;100:1281-8.

8. Chow CK, Jolly S, Rao-Melacini P, et al. Association of diet, exercise and smoking modification with risk of early cardiovascular events after acute coronary syndromes. Circulation 2010;121:750-8.

9. Rasmussen JN, Chong A, Alter DA. Relationship between adherence to evidence-based pharmacotherapy and long-term mortality after acute myocardial infarction. JAMA 2007;297:177-86.

10. Kotseva K, Wood D, De Backer G, et al. EUROASPIRE III. Management of cardiovascular risk factors in asymptomatic high-risk patients in general practice: cross-sectional survey in 12 European countries. Eur J Cardiovasc Prev Rehabil 2010;17:530-40.

11. Yusuf S, Islam S, Chow CK, et al. Use of secondary prevention drugs for cardiovascular disease in the community in high-income, middleincome, and low-income countries (the PURE Study): a prospective epidemiological survey. Lancet 2011;378:1231-43.

12. World Health Organization. Global surveillance, prevention and control of chronic respiratory diseases: a comprehensive approach. 2007. http://www.who.int/entity/gard/publications/GARD\%20Book\% 202007.pdf?ua=1

13. World Health Organization. Burden of COPD. Geneva: WHO, 2014. http://www.who.int/respiratory/copd/burden/en/

14. Briffa TG, Kinsman L, Maiorana AJ, et al. An integrated and coordinated approach to preventing recurrent coronary heart disease events in Australia. Med J Aust 2009;190:683-6.

15. Ries AL, Bauldoff GS, Carlin BW, et al. Pulmonary Rehabilitation: Joint ACCP/AACVPR Evidence-Based Clinical Practice Guidelines. Chest 2007;131(5 Suppl):4s-42.
16. Clark AM, Hartling L, Vandermeer B, et al. Meta-analysis: secondary prevention programs for patients with coronary artery disease. Ann Intern Med 2005;143:659-72.

17. Yohannes AM, Yalfani A, Doherty P, et al. Predictors of drop-out from an outpatient cardiac rehabilitation programme. Clin Rehabil 2007;21:222-9.

18. Savage PD, Sanderson BK, Brown TM, et al. Clinical research in cardiac rehabilitation and secondary prevention: looking back and moving forward. J Cardiopulm Rehabil Prev 2011;31:333-41.

19. Number of mobile phone users worldwide from 2013 to 2019 (in billions). Statistica. 2018. https://www.statista.com/statistics/274774/ forecast-of-mobile-phone-users-worldwide/ (Accessed on 27 Feb 2018).

20. Whittaker R, McRobbie H, Bullen C, et al. Mobile phone-based interventions for smoking cessation. Cochrane Database Syst Rev 2012;11:CD006611.

21. Patrick K, Raab F, Adams MA, et al. A text message-based intervention for weight loss: randomized controlled trial. J Med Internet Res 2009;11:e1.

22. Hurling R, Catt M, Boni MD, et al. Using internet and mobile phone technology to deliver an automated physical activity program: randomized controlled trial. J Med Internet Res 2007;9:e7.

23. Buis LR, Hirzel L, Turske SA, et al. Use of a text message program to raise type 2 diabetes risk awareness and promote health behavior change (part I): assessment of participant reach and adoption. J Med Internet Res 2013;15:e281.

24. Chow CK, Redfern J, Thiagalingam A, et al. Design and rationale of the tobacco, exercise and diet messages (TEXT ME) trial of a text message-based intervention for ongoing prevention of cardiovascular disease in people with coronary disease: a randomised controlled trial protocol. BMJ Open 2012;2:e000606.

25. Chow CK, Redfern J, Hillis GS, et al. Effect of Lifestyle-Focused Text Messaging on Risk Factor Modification in Patients With Coronary Heart Disease: A Randomized Clinical Trial. JAMA 2015;314:1255-63.

26. Burn E, Nghiem S, Jan S, et al. Cost-effectiveness of a text-message program for cardiovascular disease secondary prevention. Heart 2017;103:893-4.

27. Redfern J, Santo K, Coorey G, et al. Factors Influencing Engagement, Perceived Usefulness and Behavioral Mechanisms Associated with a Text Message Support Program. PLoS One 2016;11:e0163929.

28. Rennie D. CONSORT revised-improving the reporting of randomized trials. JAMA 2001;285:2006-7.

29. Thakkar J, Barry T, Thiagalingam A, et al. Design Considerations in Development of a Mobile Health Intervention Program: The TEXT ME and TEXTMEDS Experience. JMIR Mhealth Uhealth 2016;4:e127.

30. Redfern J, Thiagalingam A, Jan S, et al. Development of a set of mobile phone text messages designed for prevention of recurrent cardiovascular events. Eur J Prev Cardiol 2014;21:492-9.

31. Rasekaba T, Lee AL, Naughton MT, et al. The six-minute walk test: a useful metric for the cardiopulmonary patient. Intern Med J 2009;39:495-501.

32. Lohman TG, Roche AF, Martorell R. Anthropometric Standardization Reference Manual. Human Kinetics: Champaign, IL 1988:55-80.

33. West R, Hajek P, Stead L, et al. Outcome criteria in smoking cessation trials: proposal for a common standard. Addiction 2005;100:299-303

34. Zigmond AS, Snaith RP. The hospital anxiety and depression scale. Acta Psychiatr Scand 1983;67:361-70.

35. World Health Organisation. Global Physical Activity Questionnaire (GPAQ) Analysis Guide. 2011 http://www.who.int/chp/steps/ resources/GPAQ_Analysis_Guide.pdf (accessed 6 Jun 2017).

36. The WHO STEPwise approach to chronic disease risk factor surveillance (STEPS). Geneva: World Health Organisation, 2008. http://apps.who.int/iris/bitstream/10665/43376/1/9241593830_eng. pdf

37. Ware J, Kosinski M, Keller SD. A 12-Item Short-Form Health Survey: construction of scales and preliminary tests of reliability and validity. Med Care 1996;34:220-33.

38. Jones PW, Harding G, Berry P, et al. Development and first validation of the COPD Assessment Test. Eur Respir J 2009;34:648-54.

39. Singh SJ, Puhan MA, Andrianopoulos V, et al. An official systematic review of the European Respiratory Society/American Thoracic Society: measurement properties of field walking tests in chronic respiratory disease. Eur Respir J 2014;44:1447-78.

40. Gremeaux V, Troisgros O, Benaïm S, et al. Determining the minimal clinically important difference for the six-minute walk test and the 200-meter fast-walk test during cardiac rehabilitation program in coronary artery disease patients after acute coronary syndrome. Arch Phys Med Rehabil 2011;92:611-9. 